\title{
Estudo da conservação das propriedades nutricionais da polpa de tucumã (Astrocaryum aculeatum) in natura em embalagens a vácuo
}

\author{
Study of the conservation of the nutritional properties of in natura Tucumã pulp \\ (Astrocaryum aculeatum) using vacuum packaging
}

\author{
Sthéfanny Caroline Mendes Azevedo ${ }^{*}$, Luana Maquiné Vieira² ${ }^{2}$ Takeshi Matsuura ${ }^{3}$, Geverson Façanha da Silva², \\ Sergio Duvoisin Junior ${ }^{2}$, Patrícia Melchionna Albuquerque ${ }^{1}$ \\ 1 Universidade do Estado do Amazonas (UEA), Programa de Pós-graduação em Biotecnologia e Recursos Naturais da Amazônia, Manaus/AM - Brasil \\ 2 Universidade do Estado do Amazonas (UEA), Engenharia Química, Manaus/AM - Brasil \\ ${ }^{3}$ Universidade Federal do Amazonas (UFAM), Instituto de Ciências Biológicas, Manaus/AM - Brasil
}

\section{${ }^{*}$ Corresponding Author}

Sthéfanny Caroline Mendes Azevedo, Universidade do Estado do Amazonas (UEA), Programa de Pós-graduação em Biotecnologia e Recursos Naturais da Amazônia, Avenida Carvalho Leal, 1777, CEP: 69065-001, Manaus/AM - Brasil, e-mail: sthe.caroline90@gmail.com

Cite as: Study of the conservation of the nutritional properties of in natura Tucumã pulp (Astrocaryum aculeatum) using vacuum packaging. Braz. J. Food Technol., v. 20, e2016107, 2017.

Received: Aug. 19, 2016; Accepted: May 15, 2017

\section{Resumo}

O tucumã, também conhecido como tucumã-do-amazonas, é o fruto oriundo da palmeira Astrocaryum aculeatum e possui significativo potencial econômico local. Caracteriza-se como um fruto pouco ácido, com baixos teores de açúcar, alto teor de $\beta$-caroteno e alto valor energético. O objetivo deste trabalho foi estudar a conservação das propriedades nutricionais da polpa de tucumã in natura, a partir da utilização de embalagem a vácuo, uma vez que o armazenamento da polpa é realizado de forma inadequada, na maioria dos estabelecimentos comerciais. O potencial antioxidante da polpa durante o armazenamento também foi avaliado. Executaram-se, ao longo de 150 dias, análises de umidade, cinzas, pH, acidez, extrato etéreo, conteúdo energético, crescimento microbiológico, além da atividade antioxidante, pelo método do sequestro do radical DPPH $\bullet$. As embalagens foram armazenadas sob temperatura de congelamento e resfriamento. Foi constatado que os valores de umidade, cinzas e pH são conservados pela utilização de embalagem a vácuo, em temperatura de congelamento ou resfriamento, durante cinco meses de armazenamento; a acidez foi conservada somente em embalagens a vácuo sob congelamento, por apenas dez dias; os valores de extrato etéreo foram conservados durante três meses de congelamento e um mês de resfriamento, e o valor energético contido na polpa foi conservado por dois meses sob congelamento e dez dias sob resfriamento. Não foi detectada a presença de coliformes e de Salmonella durante cinco meses de armazenamento a vácuo, em amostras congeladas e resfriadas; a atividade antioxidante foi preservada durante dois meses em amostras congeladas, mas não foi mantida em amostras resfriadas. Portanto, a partir deste estudo, foi possível verificar que a utilização de embalagens a vácuo, associada ao congelamento, permite a conservação da maioria das principais características nutricionais da polpa de tucumã in natura, por 30 dias de armazenamento. Ressalta-se, ainda, que esse é um procedimento simples, que pode ser empregado no comércio alimentício local.

Palavras-chave: Astrocaryum aculeatum; Vácuo; Congelamento; Resfriamento; Caracterização físico-química; Atividade antioxidante.

\section{Abstract}

The tucumã, also known as Amazonian tucumã, is the fruit obtained from the Astrocaryum aculeatum palm tree, and presents significant local economic potential. It is characterized as a slightly acid fruit, low in sugar, but high in $\beta$-carotene and energy. The objective of the present work was to study the conservation of the nutritional properties of in natura tucumã pulp using vacuum packaging, since the pulp is stored inappropriately in most commercial establishments. The antioxidant potential of the pulp during storage was also evaluated. The following parameters were analysed during 150 days: 
Estudo da conservação das propriedades nutricionais da polpa de Tucumã (Astrocaryum aculeatum) in natura em embalagens a vácuo

Azevedo, S. C. M. et al.

the moisture and ash contents, pH, acidity, ether extract, energy, microbiological growth (coliforms and Salmonella), and the antioxidant activity using the DPPH• radical scavenging method. The packs were stored under freezing and refrigeration temperatures. It was found that the moisture, ash and $\mathrm{pH}$ values were retained for 5 months by the use of vacuum packaging under freezing or refrigeration temperatures; the acidity was only preserved for 10 days in vacuum packages in the freezer; the ether extract values were maintained for 3 months under freezing temperature and for 1 month under refrigeration temperature; and the energy value of the pulp was maintained for 2 months under freezing and 10 days under refrigeration temperatures. Coliforms and Salmonella were not detected during 5 months of vacuum storage under both frozen and refrigeration temperatures; and the antioxidant activity was preserved for 2 months in the frozen samples, but not in the refrigerated samples. Thus this study showed that the use of vacuum packaging associated with freezing conserved the majority of the nutritional properties of the in in natura tucumã pulp for 30 days of storage, a simple procedure that can be used by local food suppliers.

Keywords: Astrocaryum aculeatum; Vacuum; Freezing; Cooling; Physicochemical characterisation; Antioxidant activity.

\section{Introdução}

A Amazônia é rica em frutas que possuem potencial econômico local (BRAGA et al., 2010). Entre estas, destaca-se o tucumã, também conhecido como tucumã-do-amazonas, tucumã-açu e jabarana (COSTA et al., 2005), caracterizado como um fruto suculento, pouco ácido, com baixos teores de açúcar, alto de teor de $\beta$-caroteno e alto valor energético (YUYAMA et al., 2008). O fruto é oriundo da palmeira Astrocaryum aculeatum, que está distribuída na região central da bacia amazônica, sendo, no Brasil, encontrada nos Estados de Amazonas, Acre, Pará, Rondônia e Roraima (KAHN, 2008).

O tucumã é comercializado in natura, em dúzia ou cento, ou processado pelos comerciantes locais, sendo sua polpa vendida por peso. De abril de 2011 a maio de 2012, o valor médio da saca de tucumã vendida nas feiras da cidade de Manaus, no atacado, foi de 80 reais, e, no varejo, o valor médio da dúzia foi de três reais e do quilo da polpa, 30 reais, sendo os principais fornecedores do fruto os municípios de Itacoatiara-AM, Autazes-AM, Rio Preto da Eva-AM, Terra Santa-PA, Barreirinha-AM e Careiro Castanho-AM (DIDONET; FERRAZ, 2014). Comumente, a polpa do tucumã é consumida in natura, acompanhada de farinha de mandioca, em sanduíche de tucumã e na forma de sucos e sorvete (MOUSSA; KAHN, 1997; KAHN, 2008), sendo o comércio do fruto diretamente ligado ao surgimento de estabelecimentos que têm produtos alimentícios tradicionais da região como principal atrativo (CLEMENT et al., 2005).

Ao se obter a polpa de tucumã, a mesma deve ser consumida em um dia, sob temperatura ambiente, ou em uma semana, sob refrigeração (SCHROTH et al., 2004). Porém, a realidade verificada no comércio da polpa do fruto é bem diferente do que é recomendado por estudos científicos. Na cidade de Manaus, é possível verificar a polpa de tucumã sendo armazenada de maneira inadequada, o que contribui para a proliferação de microrganismos e o desenvolvimento de doenças transmitidas por alimentos.
A legislação brasileira prevê os padrões microbiológicos sanitários para polpas de frutas, concentradas ou não, com ou sem tratamento térmico, refrigeradas ou congeladas, tendo como tolerância um número mais provável (NMP) de $10^{5}$ coliformes a $45^{\circ} \mathrm{C}$ por grama (g) de polpa e ausência de Salmonella spp. em $25 \mathrm{~g}$, sendo uma condição sanitária satisfatória aquela em que os resultados se encontram abaixo ou iguais àqueles pré-estabelecidos (BRASIL, 2001).

O emprego de vácuo em embalagens promove a supressão do oxigênio, o que tende a aumentar a vida útil dos alimentos, uma vez que retarda a respiração, o amadurecimento, o envelhecimento, a perda da umidade, a modificação da textura, o escurecimento enzimático e o desenvolvimento de microrganismos (CENCl, 2011). A utilização de embalagem a vácuo se mostra eficiente na conservação de carnes, massas, ovos e grãos (HERNÁNDEZ-MACEDO et al., 2011; PRENTICE; SAINZ, 2005; CRUZ et al., 2006; SCATOLINI-SILVA et al., 2013; LIMA et al., 2014).

Levando-se em consideração que o tucumã possui comprovado potencial econômico, bem como ótimas características nutricionais, neste trabalho, foi avaliada a conservação de suas propriedades nutricionais, a atividade antioxidante e o crescimento microbiológico, em embalagens a vácuo, sob congelamento e resfriamento, durante 150 dias.

\section{Materiais e métodos}

\subsection{Coleta dos frutos e obtenção da polpa}

Frutos de tucumã foram coletados durante a época de safra (abril de 2015), em um sítio situado no município de Rio Preto da Eva-AM (2³7'31,8"S 5944'52,6"O), conforme proposto por Ramos et al. (2009). A polpa foi obtida executando-se a metodologia proposta por Flor et al. (2015). 
Estudo da conservação das propriedades nutricionais da polpa de Tucumã (Astrocaryum aculeatum) in natura em embalagens a vácuo

Azevedo, S. C. M. et al.

\subsection{Armazenamento}

Com a polpa total obtida, procedeu-se à mistura da mesma, a fim de se obter uma amostra homogênea. Posteriormente, houve a separação da polpa, produzindo-se 14 embalagens a vácuo, em sacos de filme multicamada à base de poliamida e polietileno, contendo polpa de tucumã, cada uma pesando $200 \mathrm{~g}$. Destas, sete embalagens foram armazenadas sob resfriamento $\left(5^{\circ} \mathrm{C}\right)$ e sete sob congelamento $\left(-18^{\circ} \mathrm{C}\right)$.

\subsection{Caracterização físico-química, análise microbiológica e atividade antioxidante}

Imediatamente e após 10, 30, 60, 90, 120 e 150 dias, foram realizadas as análises de umidade, cinzas, $\mathrm{pH}$, acidez e lipídios, conforme metodologia do IAL (2008), e do conteúdo energético, por calorimetria (ABNT, 1984), utilizando-se calorímetro IKA ${ }^{\circledR}$ C200. A presença de coliformes a $45^{\circ} \mathrm{C}$ e Salmonella spp. foi verificada através da técnica de membrana filtrante proposta pela CETESB (1993, 2007). A análise da atividade antioxidante foi realizada através da técnica de sequestro do DPPH•, desenvolvida por Brand-Williams et al. (1995) e modificada por Duarte-Almeida et al. (2006) e por Silva (2012).

\subsubsection{Atividade antioxidante}

Para obtenção das amostras, foram pesados 7,91 g de polpa de tucumã, os quais foram macerados com almofariz e pistilo e solubilizados em $50 \mathrm{~mL}$ de metanol P.A. (proporção de 1:5 m/v), em triplicata. Após seis dias, o extrato foi filtrado e a concentração foi determinada adicionando-se $5 \mathrm{~mL}$ da solução metanólica obtida a uma placa de Petri previamente tarada, evaporando o metanol em estufa a $40{ }^{\circ} \mathrm{C}$, até peso constante. A solução de $\mathrm{DPPH} \bullet$ foi preparada na concentração de 0,06 mmol/L, com metanol P.A., no mesmo dia do ensaio da atividade antioxidante, sendo a solução protegida da exposição direta da luz.

O ensaio foi realizado através de espectrofotometria em microplaca, diluindo o filtrado com metanol, em progressão geométrica, na razão de 0,5, até a obtenção de diluição de 512 vezes, com volume de $40 \mu \mathrm{L}$ em cada diluição, e adição de $250 \mu \mathrm{L}$ da solução de DPPH•. Para o controle, foram adicionados $40 \mu \mathrm{L}$ de metanol e $250 \mu \mathrm{L}$ da solução de DPPH•, e para o branco, $40 \mu \mathrm{L}$ de cada diluição e $250 \mu \mathrm{L}$ da solução de metanol. A microplaca foi protegida da exposição direta da luz e, após 25 minutos, as leituras de absorbância foram realizadas em espectrofotômetro de microplaca (Molecular Devices, modelo Spectramax Plus), a $517 \mathrm{~nm}$. Ácido ascórbico e quercetina foram utilizados como padrões, nas concentrações de 20, 16, 12, 8, 4, e $0 \mu \mathrm{g} / \mathrm{mL}$.

A porcentagem do sequestro de radicais DPPH• foi medida através da Equação 1, utilizando os valores dos decaimentos de absorbância da amostra (Absamostra) e do controle (Abscontrole):

$$
\mathrm{AA} \%=\frac{(\text { Abscontrole }- \text { Absamostra })}{\text { Abscontrole }} \times 100
$$

Foi possível ser determinada, a partir da geração de um gráfico de regressão linear, a concentração de amostra de extrato da polpa de tucumã necessária para sequestro de $50 \%$ dos radicais DPPH $\bullet$, determinando-se, assim, a Concentração Eficiente $\left(\mathrm{CE}_{50}\right)$.

\subsection{Análise estatística}

Os resultados das análises físico-químicas e da atividade antioxidante da polpa de tucumã foram submetidos à análise de variância ANOVA, para amostras homogêneas, e ao teste de Tukey, para distinguir as diferenças, levando-se em consideração o intervalo de confiança de $95 \%$. Para comparação entre os dois tratamentos, foi utilizado o teste T. Os dados que apresentaram diferenças significativas em função do tempo de armazenamento foram avaliados quanto à correlação, avaliação esta seguida de regressão linear. O software utilizado foi o BioEstat 5.0.

\section{Resultados e discussão}

\subsection{Caracterização físico-química}

A variação das características fisico-químicas da polpa de tucumã embalada a vácuo, sob congelamento e resfriamento, encontra-se descrita nas Tabelas 1 e 2, respectivamente.

Nas amostras congeladas, foi observada a conservação do valor de umidade inicial durante dois meses de armazenamento, não havendo diferença estatística durante o período de dez dias a cinco meses (Tabela 1). Para as amostras resfriadas, não houve variação da umidade nos cinco meses de armazenamento (Tabela 2). Comparando-se os dois tratamentos, não foram constatadas diferenças estatísticas significativas $(p<0,05)$. Foi possível verificar que a embalagem a vácuo preservou a umidade da polpa de tucumã, ocorrendo uma pequena variação dessa propriedade no armazenamento da polpa sob congelamento. Contudo, a ausência de diferença entre os tratamentos pressupõe que as temperaturas de congelamento e resfriamento não diferem na conservação da umidade da polpa de tucumã, quando são utilizadas embalagens a vácuo. Os coeficientes de determinação de 0,56 e 0,60, obtidos para os tratamentos sob congelamento e resfriamento, respectivamente, indicam, entretanto, que outros fatores, não avaliados neste estudo, influenciam na umidade da polpa de tucumã.

A água contida nos alimentos está ligada à ocorrência de diversas reações químicas, físicas e microbiológicas, e valores baixos de umidade estão relacionados ao aumento de vida útil de alimentos (VASCONCELOS; MELO FILHO, 
Estudo da conservação das propriedades nutricionais da polpa de Tucumã (Astrocaryum aculeatum) in natura em embalagens a vácuo

Azevedo, S. C. M. et al.

Tabela 1. Caracterização físico-química de polpa de tucumã in natura armazenada em embalagem a vácuo sob congelamento.

\begin{tabular}{|c|c|c|c|c|c|c|c|c|}
\hline \multirow{2}{*}{ Componentes } & \multicolumn{7}{|c|}{ Tempo de Armazenamento (dias) } & \multirow{2}{*}{$\mathbf{R}^{2}$} \\
\hline & 0 & 10 & 30 & 60 & 90 & 120 & 150 & \\
\hline Umidade (\%) & $52,54 \pm 3,32^{A}$ & $58,20 \pm 0,66^{A B}$ & - & $58,78 \pm 2,48 \mathrm{AB}$ & $64,07 \pm 2,33^{B}$ & $62,38 \pm 2,32^{B}$ & $60,70 \pm 0,97^{B}$ & 0,5629 \\
\hline Cinzas (\%) & $1,08 \pm 0,05^{c}$ & $1,01 \pm 0,02^{c}$ & - & - & - & $1,12 \pm 0,00^{c}$ & $1,00 \pm 0,05^{c}$ & 0,0002 \\
\hline $\mathrm{pH}$ & $5,2 \pm 0,02 \mathrm{D}$ & $5,33 \pm 0,05^{D}$ & - & $5,34 \pm 0,13^{D}$ & $5,44 \pm 0,00^{D}$ & $5,31 \pm 0,04^{D}$ & $5,19 \pm 0,00^{D}$ & 0,0006 \\
\hline Acidez (\%) & $0,64 \pm 0,00 \mathrm{E}$ & $0,72 \pm 0,00 \mathrm{EF}$ & $0,95 \pm 0,00^{F}$ & - & - & $1,31 \pm 0,16^{G}$ & $1,67 \pm 0,00^{G}$ & 0,9654 \\
\hline Extrato etéreo $(\%)$ & $21,25 \pm 1,72 \mathrm{~J}$ & $20,11 \pm 1,58$ & - & $21,88 \pm 0,00^{J}$ & $22,17 \pm 0,00^{\jmath}$ & $13,77 \pm 0,00^{k}$ & $13,34 \pm 0,48^{k}$ & 0,543 \\
\hline Energia (kcal/g) & $12,32 \pm 0,00^{P}$ & - & - & $11,54 \pm 0,01^{Q}$ & $11,30 \pm 0,00^{R}$ & $10,18 \pm 0,00^{s}$ & $9,66 \pm 0,00^{\top}$ & 0,9417 \\
\hline
\end{tabular}

Letras diferentes na mesma linha indicam diferença estatística no nível de 5\%; - Análise não realizada; $\mathrm{R}^{2}$ coeficiente de determinação obtido por meio de regressão linear dos valores obtidos em função do tempo de armazenamento.

Tabela 2. Caracterização físico-química de polpa de tucumã in natura armazenada em embalagem a vácuo sob resfriamento.

\begin{tabular}{|c|c|c|c|c|c|c|c|c|}
\hline \multirow{2}{*}{ Componentes } & \multicolumn{7}{|c|}{ Tempo de Armazenamento (dias) } & \multirow{2}{*}{$\mathbf{R}^{2}$} \\
\hline & 0 & 10 & 30 & 60 & 90 & 120 & 150 & \\
\hline Umid & $52,54 \pm 3,32^{A}$ & - & - & $59,99 \pm 1,21^{A}$ & $60,56 \pm 1,54^{A}$ & $59,58 \pm 4,68^{A}$ & $59,77 \pm 2,47^{A}$ & 0,6036 \\
\hline Cinzas (\%) & $1,08 \pm 0,05^{c}$ & $1,00 \pm 0,05^{c}$ & - & - & $0,86 \pm 0,06^{c}$ & $1,12 \pm 0,00^{c}$ & $1,04 \pm 0,08^{c}$ & 0,0002 \\
\hline $\mathrm{pH}$ & $5,2 \pm 0,00^{D}$ & - & - & $5,36 \pm 0,06^{D}$ & - & $5,34 \pm 0,06^{D}$ & $5,24 \pm 0,04^{D}$ & 0,0796 \\
\hline Acidez (\%) & $0,64 \pm 0,00 \mathrm{E}$ & $0,72 \pm 0,00 \mathrm{~F}$ & $0,95 \pm 0,00^{G}$ & $1,67 \pm 0,00^{H}$ & - & $1,90 \pm 0,00^{\prime}$ & $1,89 \pm 0,01$ & 0,8711 \\
\hline Extrato etéreo (\%) & $21,25 \pm 1,72^{\jmath}$ & $17,10 \pm 1,30 \triangleleft$ & $15,59 \pm 0,00^{\llcorner}$ & - & - & $0,84 \pm 0,56^{M}$ & - & 0,9859 \\
\hline Energia (kcal/g) & $12,32 \pm 0,00^{u}$ & $11,69 \pm 0,14^{v}$ & - & $11,97 \pm 0,00^{v}$ & - & $11,31 \pm 0,00 w$ & $9,09 \pm 0,00 x$ & 0,6879 \\
\hline
\end{tabular}

Letras diferentes na mesma linha indicam diferença estatística no nível de 5\%; - Análise não realizada; $\mathrm{R}^{2}$ coeficiente de determinação obtido por meio de regressão linear dos valores obtidos em função do tempo de armazenamento.

2010). Neste sentido, Lima (2013) também verificou, através da utilização de embalagens a vácuo, a conservação dos valores de umidade em grãos de feijão, durante oito meses de armazenamento, o que colaborou para o aumento da vida-de-prateleira do alimento em questão, podendo-se verificar que tais embalagens formam uma barreira, minimizando trocas gasosas entre o alimento embalado e o ambiente exterior.

Neste estudo, não houve variação nos valores de cinzas ao longo do período de armazenamento das amostras congeladas (Tabela 1) e das amostras resfriadas (Tabela 2). Ainda, entre os dois tratamentos, também não foi constatada diferença estatística, podendo ser inferido que as temperaturas de congelamento e resfriamento não interferem na conservação da quantidade de cinzas da amostra de tucumã, quando utilizada a embalagem a vácuo. Os baixos coeficientes de correlação obtidos confirmam o fato de não haver correlação entre a porcentagem de cinzas da polpa de tucumã e o tempo de armazenamento sob diferentes temperaturas. Os valores obtidos para a quantidade de cinzas estão de acordo com os reportados na literatura. Yuyama et al. (2008) encontraram 1,26\% de cinzas na polpa de tucumã in natura, enquanto Flor et al. (2015) encontraram 1,9 g/100 g.

Alimentos com pH acima de 4,5 são considerados pouco ácidos, como é o caso da polpa de tucumã. Paralelamente, a maioria dos microrganismos associados a alimentos cresce na faixa de pH de 5 a 8 (AZEREDO, 2012). Com isso, observa-se a importância de se conservarem os valores de $\mathrm{pH}$ em alimentos, a fim de que não haja proliferação de microrganismos patogênicos ou que estes causem deterioração nos mesmos. Desta forma, a conservação a vácuo se mostrou eficiente na conservação do pH da polpa de tucumã, uma vez que não houve variação nos valores de pH entre os períodos de armazenamento nas amostras congeladas (Tabela 1), bem como nas amostras resfriadas (Tabela 2). Da mesma forma, os coeficientes de determinação encontrados foram baixos, corroborando a ausência de correlação do $\mathrm{pH}$ com o tempo de armazenamento. Ainda, entre os dois tratamentos, também não foi constatada diferença neste parâmetro, podendo-se afirmar que as temperaturas de congelamento e resfriamento, assim como na análise de cinzas, não interferem na conservação do valor de $\mathrm{pH}$ da amostra de tucumã, quando utilizada a embalagem a vácuo. De maneira semelhante, Silva et al. (2010) não observaram alteração dos valores de $\mathrm{pH}$ em polpa de frutos de bacuri (Platonia insignis Mart.), por 360 dias, em embalagem a vácuo, sob congelamento. Em outro estudo, o pH de laranjas Champagne sob refrigeração também não apresentou diferenças significativas ao longo de 60 dias de armazenamento (AGOSTINI et al., 2014).

Em um processo de decomposição por hidrólise, oxidação ou fermentação, é comum a alteração da concentração de íons de hidrogênio, sendo a determinação de acidez fundamental na avaliação da conservação de um alimento (IAL, 2008). A variação de acidez em amostras de polpa de tucumã congeladas mostra que as embalagens a vácuo associadas à temperatura de congelamento permitiram a conservação do valor de acidez até 30 dias 
de armazenamento (Tabela 1). Esse resultado está de acordo com o encontrado por Rinaldi et al. (2015), para o congelamento de raízes de mandioca a $-18{ }^{\circ} \mathrm{C}$, que se mostrou eficiente na conservação da acidez titulável, durante 31 dias de armazenamento.

As embalagens a vácuo armazenadas sob temperatura de resfriamento não conservaram a porcentagem inicial de acidez durante quatro meses de armazenamento, porém a variação de acidez entre quatro e cinco meses de armazenamento não apresentou diferença significativa. Comparando-se os dois tratamentos, verificou-se que, até um mês de armazenamento, as temperaturas de congelamento e resfriamento, em conjunto com o processamento a vácuo, produzem o mesmo efeito sobre a conservação da acidez em polpas de tucumã, não conseguindo, entretanto, manter os mesmos valores de acidez iniciais da polpa recém-coletada. Porém, após quatro meses de armazenamento, verificou-se que as embalagens a vácuo armazenadas sob congelamento forneceram valores de acidez inferiores aos das embalagens armazenadas sob resfriamento. Com isso, foi possível constatar que, ainda que não tenha conservado o valor de acidez inicial da polpa de tucumã, a associação dos tratamentos de congelamento e acondicionamento a vácuo se mostra superior ao de resfriamento e embalagem a vácuo. Os altos valores de $R^{2}$ observados (superiores a 0,85 ) indicam a existência de correlação entre a acidez e o tempo de armazenamento, mostrando que as alterações nos índices de acidez são proporcionais ao tempo de armazenamento e que esta propriedade pode ser levemente retardada sob congelamento. Esse fato ocorre porque, sob baixas temperaturas, a energia livre do sistema é menor, diminuindo a velocidade de degradação ou oxidação de lipídios, o que influencia diretamente no índice de acidez (SILVA et al., 1999).

A embalagem a vácuo associada à temperatura de congelamento conservou a porcentagem de extrato etéreo da polpa de tucumã durante três meses de armazenamento, decaindo em 1,5 vez após quatro meses (Tabela 1), enquanto que a embalagem a vácuo associada à temperatura de resfriamento não garantiu conservação da porcentagem do extrato etéreo. Tal fato se deu, provavelmente, devido à ocorrência de degradação lipídica e consequente alteração no conteúdo energético após um mês de armazenamento, sendo constatada uma diminuição gradativa em tais valores, havendo decaimento de 1,9 vez, após quatro meses de armazenamento (Tabela 2). Na comparação das duas temperaturas de armazenamento, verificou-se que, até dez dias, tais tratamentos comportaram-se da mesma maneira; porém, após quatro meses, o armazenamento sob congelamento se mostrou mais eficiente. Também foi possível verificar que, sob congelamento, o efeito do tempo de armazenamento sobre o conteúdo lipídico diminuiu $\left(R^{2}=0,54\right)$, quando comparado com as amostras sob resfriamento $\left(R^{2}=0,99\right)$. Sabendo-se que os lipídios são compostos orgânicos altamente energéticos, contendo ácidos graxos essenciais ao organismo, atuando como transportadores das vitaminas lipossolúveis (IAL, 2008), ressalta-se a importância da conservação desta característica físico-química da polpa de tucumã, uma vez que o significativo conteúdo energético na mesma está diretamente relacionado ao extrato etéreo.

A partir da análise das amostras congeladas, foi possível observar que não houve conservação do conteúdo energético da polpa de tucumã, após dois meses de congelamento. Os valores energéticos decaíram 1,27 vez (Tabela 1) após cinco meses de armazenamento. Nas amostras resfriadas, foi possível verificar o decaimento significativo dos valores energéticos após dez dias de armazenamento e, após cinco meses de armazenamento, houve queda de 1,35 vez. Os dados de correlação linear mostram que o conteúdo energético variou significativamente em função do tempo de armazenamento, ou seja, esta propriedade não foi influenciada pelas temperaturas de armazenamento avaliadas. De acordo com a literatura, o conteúdo energético da polpa de tucumã encontra-se entre 3,62 e 3,33 kcal/g (YUYAMA et al., 2008; FLOR et al., 2015). Tais valores foram estimados a partir das análises de lipídios, proteínas e carboidratos. Contudo, neste trabalho, foram encontrados valores de até 3,4 vezes maiores que os da literatura, uma vez que foram calculados através da utilização de calorímetro. Tal discrepância foi relatada por Santos (2010), verificando que a análise do conteúdo energético de alimentos sólidos, realizada através de calorímetro, permite determinar um poder calorífico superior à registrada nos rótulos de tais alimentos, valores estes comumente estimados através da composição nutricional. O decaimento do conteúdo energético observado nas amostras congeladas e resfriadas relaciona-se com o decaimento dos extratos etéreos nos dois tipos de armazenamento, uma vez que os lipídeos são os principais responsáveis pela energia da polpa de tucumã (YUYAMA et al., 2008).

\subsection{Presença de Coliformes e Salmonella}

Os ensaios para verificação da presença de coliformes e de Salmonella em amostras de polpa de tucumã congeladas e resfriadas indicaram ausência desses microrganismos em todas as amostras.

A ausência de coliformes totais, coliformes a $45^{\circ} \mathrm{C}$ e Salmonella nas amostras mostra que a polpa de tucumã embalada a vácuo, sob congelamento e resfriamento, após cinco meses de armazenamento, apresenta qualidade microbiológica, de acordo com os atuais padrões nacionais de identidade e qualidade para polpa de frutas, de forma semelhante ao que foi verificado por Santos et al. (2010). No entanto, durante as análises para detecção de coliformes e Salmonella, foi observada proliferação 
Estudo da conservação das propriedades nutricionais da polpa de Tucumã (Astrocaryum aculeatum) in natura em embalagens a vácuo

Azevedo, S. C. M. et al.

de grande quantidade de outros microrganismos, com características diferentes destes analisados.

É possível que a polpa de tucumã contenha substâncias nocivas a coliformes e Salmonella, porém inertes a estes outros microrganismos observados. Deve-se, dessa forma, ser realizado um estudo aprofundado a respeito da identificação destes microrganismos presentes na polpa de tucumã, a fim de que seja verificada a viabilidade da polpa in natura, armazenada em embalagens a vácuo, para fins alimentícios.

A heterogeneidade do efeito antimicrobiano da polpa de tucumã foi observada por Jobim et al. (2014), em que o extrato etanólico da polpa apresentou atividade contra E. faecalis, B. cereus, L. monocytogenese e C. albicans, ao mesmo tempo em que não foi detectada atividade contra outras dezenas de microrganismos. Tal especificidade antimicrobiana foi associada à rica composição química da polpa, contendo grande quantidade de compostos fenólicos, o que pode variar conforme o local e o período de coleta, bem como com a maturação dos frutos (MONTEIRO et al., 2005).

A utilização de embalagens a vácuo no combate à proliferação de coliformes e Salmonella vem sendo reportada na literatura. Dias (2011) verificou que, sob resfriamento, o uso de embalagens a vácuo para armazenar batatas palito resultou em boa qualidade microbiológica frente a coliformes e Salmonella, durante 12 dias de armazenamento. O armazenamento a vácuo também se mostrou eficiente na conservação de massas frescas de lasanha, sem aditivos, controlando o crescimento de fungos filamentosos, leveduras, Staphylococcus spp., coliformes totais e Escherichia. coli, sob temperatura de $10 \pm 2{ }^{\circ} \mathrm{C}$, por até 30 dias de armazenamento (CRUZ et al., 2006). Porém, estudos relatam que a utilização de recipientes com vácuo aumenta o risco do desenvolvimento de metabolismo anaeróbico de microrganisnos, sendo necessários estudos aprofundados sobre tal problemática (KNEE; AGGARWAL, 2000).

\subsection{Atividade antioxidante}

A partir das análises de atividade antioxidante, foi possível verificar que o extrato da polpa de tucumã apresentou atividade antioxidante 8,65 vezes menos eficiente $\left(C E_{50}=154,34 \mu \mathrm{g} / \mathrm{mL}\right)$ que o padrão quercetina $\left(\mathrm{CE}_{50}=17,83 \mu \mathrm{g} / \mathrm{mL}\right)$ e 7,96 vezes menos eficiente que o padrão ácido ascórbico $\left(\mathrm{CE}_{50}=19,37 \mu \mathrm{g} / \mathrm{mL}\right)$, o que pode ser observado nas Figuras 1 e 2.

Em amostras congeladas, a atividade antioxidante foi mantida até dois meses de armazenamento, com diminuição de 1,06 vez, após três meses. Também se verificou que não houve diferença estatística entre a atividade antioxidante da polpa armazenada entre três e cinco meses, com redução de apenas 1,61 vez, após cinco meses de armazenamento (Figura 1). Diferentemente, nas amostras resfriadas, observou-se variação na atividade antioxidante a partir de dez dias de armazenamento, com queda de 1,44 vez. Após os cinco meses de armazenamento, a atividade antioxidante da polpa resfriada diminuiu 7,65 vezes (Figura 2).

A embalagem a vácuo utilizada não foi determinante na conservação da atividade antioxidante da polpa de tucumã, uma vez que a sua associação com a temperatura de resfriamento não forneceu proteção desta propriedade, ao contrário do que foi verificado no armazenamento sob congelamento. A conservação da atividade antioxidante através do congelamento de polpas de frutas também foi verificada por Freire et al. (2013). Os autores armazenaram a polpa de acerola (Malpighia emarginata) sob temperatura de $-18^{\circ} \mathrm{C}$ e verificaram que a atividade antioxidante se manteve inalterada durante três meses de armazenamento. Manach et al. (2004) afirmaram que a concentração de compostos fenólicos, principais responsáveis pela atividade antioxidante em plantas, pode ser afetada por fatores, como processamento, armazenamento, bem como por fatores ambientais diversos. Na polpa de tucumã, já

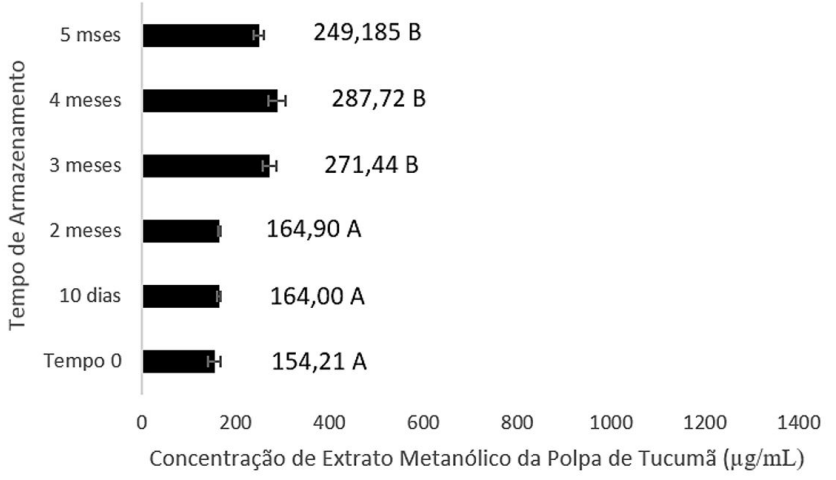

Figura 1. Variação da Concentração Eficiente a 50\% $\left(\mathrm{CE}_{50}\right)$ em polpa de tucumã congelada. Letras diferentes indicam diferença estatística no nível de 5\%.

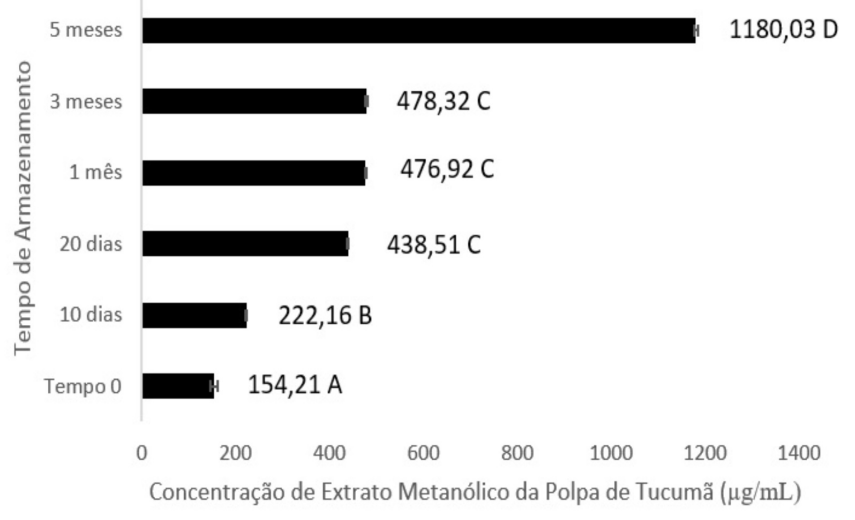

Figura 2. Variação da Concentração Eficiente a 50\% $\left(\mathrm{CE}_{50}\right)$ em polpa de tucumã resfriada. Letras diferentes indicam diferença estatística no nível de $5 \%$. 
Estudo da conservação das propriedades nutricionais da polpa de Tucumã (Astrocaryum aculeatum) in natura em embalagens a vácuo

Azevedo, S. C. M. et al.

foi observada a presença de flavonoides, como rutina e quercetina, e de taninos, como ácido gálico, ácido cafeico e ácido clorogênico.

A redução da atividade antioxidante verificada nas amostras de polpa de tucumã embaladas a vácuo sob refrigeração indica, portanto, que esta temperatura pode interferir na concentração de compostos fenólicos, prováveis responsáveis pela atividade antioxidante da polpa de tucumã. Nota-se também que a embalagem a vácuo utilizada associada ao congelamento pode conservar a concentração de compostos fenólicos por mais tempo de armazenamento e, consequentemente, a atividade antioxidante da polpa de tucumã.

Levando-se ainda em consideração a verificação da proliferação de uma grande quantidade de microrganismos não identificados, durante a análise de presença de coliformes e Salmonella, é possível inferir que exista relação entre tais microrganismos e a degradação de compostos fenólicos, como relatado por Zanin et al. (2014).

\section{Conclusão}

As propriedades que se mantiveram conservadas na polpa de tucumã in natura ao longo dos 150 dias de armazenamento, em embalagens a vácuo, foram teor de cinzas e pH. A associação de embalagem a vácuo e temperatura de congelamento mostrou-se mais eficiente na conservação da acidez, do extrato etéreo e, consequentemente, do conteúdo energético.

A utilização de embalagem a vácuo não permitiu a proliferação de coliformes e Salmonella, durante 150 dias de armazenamento, sob congelamento ou resfriamento; porém, pode induzir o crescimento de outros microrganismos, necessitando tal ocorrência de mais estudos. A presença de uma grande quantidade de microrganismos na polpa de tucumã armazenada pode estar relacionada ao pH da polpa, que permite a proliferação de bactérias e fungos diversos.

A associação da embalagem a vácuo com armazenamento sob congelamento conserva a atividade antioxidante da polpa de tucumã, havendo pouca redução de atividade ao longo de cinco meses de armazenamento. O período de armazenamento que preservou todas as propriedades nutricionais estudadas da polpa de tucumã foi de 30 dias, sob vácuo e congelamento. Este procedimento pode ser empregado por estabelecimentos alimentícios, que comercializam a polpa de tucumã, a fim de manter sua qualidade nutricional por mais tempo.

\section{Referências}

AGOSTINI, J. S.; SCALON, S. P. Q.; LESCANO, C. H.; SILVA, K. E.; GARCETE, G. J. Nota científica: conservação pós-colheita de laranjas Champagne (Citrus reticulata $\times$ Citrus sinensis). Brazilian Journal of Food Technology, v. 17, n. 2, 2014.
ASSOCIAÇÃO BRASILEIRA DE NORMAS TÉCNICAS - ABNT. NBR 8633: carvão vegetal: determinação do poder calorífico. Rio de Janeiro, 1984. 13 p.

AZEREDO, H. M. C. Fundamentos de estabilidade de alimentos. 2. ed. Brasília, DF: EMBRAPA, 2012. 326 p.

BRAGA, A. C. C.; SILVA, A. E.; PELAIS, A. C. A.; BICHARA, C. M. G.; POMPEU, D. R. Atividade antioxidante e quantificação de compostos bioativos dos frutos de abricó (Mammea americana).

Alimentos e Nutrição, v. 21, n. 1, p. 31-36, 2010.

BRAND-WILLIAMS, W.; CUVELIER, M. E.; BERSET, C. Use of free radical method to evaluate antioxidant activity. LebensmittelWissenschaft + Technologie, v. 28, n. 1, p. 25-30, 1995. http:// dx.doi.org/10.1016/S0023-6438(95)80008-5.

BRASIL. Agência Nacional de Vigilância Sanitária. Resolução RDC $n^{\circ} 12$, de 2 de janeiro de 2001. Aprova o Regulamento Técnico sobre padrões microbiológicos para alimentos. Diário Oficial [da] República Federativa do Brasil, Brasília, DF, 2001. 48 p.

CENCI, S. A. Processamento mínimo de frutas e hortaliças: tecnologia, qualidade e sistemas de embalagem. Rio de Janeiro: Embrapa Agroindústria de Alimentos, 2011. 144 p.

CLEMENT, C. R.; LLERAS PÉREZ, E.; LEEUWEN, J. O potencial das palmeiras tropicais do Brasil: acertos e fracassos das últimas décadas. Agrociências, v. 9, n. 1, p. 67-71, 2005.

COMPANHIA DE TECNOLOGIA DE SANEAMENTO AMBIENTAL DE SÃO PAULO - CETESB. Salmonella: isolamento e identificação: método de ensaio. São Paulo: CETESB, 1993. 42 p.

COMPANHIA DE TECNOLOGIA DE SANEAMENTO AMBIENTAL DE SÃO PAULO - CETESB. Coliformes totais: determinação pela técnica de membrana filtrante. São Paulo: CETESB, 2007. 55 p.

COSTA, J. R.; LEEUWEN, J.; COSTA, J. A. Tucumã do Amazonas: Astrocaryum tucuma Martinus. In: SHANLEY, P.; MEDINA, G. Frutíferas e plantas úteis na vida amazônica. Belém: CIFOR \& lamazon, 2005. cap. 2, p. 209-222.

CRUZ, R. S.; SOARES, N. F. F.; ANDRADE, N. J. Evaluation of oxygen absorber on antimicrobial preservation of lasagna-type fresh pasta under vacuum packed. Ciência e Agrotecnologia, v. 30, n. 6, p. 1135-1138, 2006.

DIAS, P. D. B. Efeito da utilização de película e diferentes formas de acondicionamento na conservação pós-colheita e fritura de batatas minimamente processadas. 2011. $137 \mathrm{f}$. Dissertação (Mestrado em Ciência e Tecnologia de Alimentos)Escola Superior de Agricultura "Luiz de Queiroz", Universidade de São Paulo, Piracicaba, 2011.

DIDONET, A. A.; FERRAZ, I. D. K. O comércio de frutos de tucumã (Astrocaryum aculeatum G. Mey - Arecaceae) nas feiras de Manaus (Amazonas, Brasil). Revista Brasileira de Fruticultura, v. 36, n. 2, p. 353-362, 2014.

DUARTE-ALMEIDA, J. M.; SANTOS, R. J.; GENOVESE, M. I.; LAJOLO, F. M. Avaliação da atividade antioxidante utilizando 
Estudo da conservação das propriedades nutricionais da polpa de Tucumã (Astrocaryum aculeatum) in natura em embalagens a vácuo

Azevedo, S. C. M. et al.

$\beta$-caroteno/ácido linoléico e método de sequestro de radicais DPPH. Ciência e Tecnologia de Alimentos, v. 26, n. 2, p. 446-452, 2006.

FLOR, N. S.; ANDRADE, J. S.; FERREIRA, S. A. N. Nutritional value and sensorial attributes of tucum paste. British Journal of Applied Science and Technology, v. 5, n. 4, p. 371-379, 2015. http://dx.doi.org/10.9734/BJAST/2015/13160.

FREIRE, J. M.; ABREU, C. M. P.; ROCHA, D. A.; CORRÊA, A. D.; MARQUES, N. R. Quantificação de compostos fenólicos e ácido ascórbico em frutos e polpas congeladas de acerola, caju, goiaba e morango. Ciência Rural, v. 43, n. 12, p. 2291-2296, 2013.

HERNÁNDEZ-MACEDO, M. L.; BARANCELLI, G. V.; CONTRERASCASTILLO, C. J. Microbial deterioration of vacuum-packaged chilled beef cuts and techniques for microbiota detection and characterization: a review. Brazilian Journal of Microbiology, v. 42, n. 1, p. 1-11, 2011. http://dx.doi.org/10.1590/S151783822011000100001.

INSTITUTO ADOLFO LUTZ - IAL. Normas Analíticas do Instituto Adolfo Lutz: métodos Físico-químicos para análise de alimentos. 4. ed. São Paulo: IAL, 2008. 1020 p.

JOBIM, M. L.; SANTOS, R. C.; ALVES, C. F. S.; OLIVEIRA, R. M.; MOSTARDEIRO, C. P.; SAGRILLO, M. R.; SOUZA FILHO, O. C.; GARCIA, L. F. M.; MANICA-CATTANI, M. F.; RIBEIRO, E. E.; CRUZ, I. B. M. Antimicrobial activity of Amazon Astrocaryum aculeatum extracts and its association to oxidative metabolism. Microbiological Research, v. 169, n. 4, p. 314-323, 2014. http:// dx.doi.org/10.1016/j.micres.2013.06.006.

KAHN, F. The genus Astrocaryum (Arecaceae). Revista Peruana de Biología, v. 15, n. 1, p. 31-48, 2008.

KNEE, M.; AGGARWAL, D. Evaluation of vacuum containers for consumer storage of fruits and vegetables. Postharvest Biology and Technology, v. 19, n. 1, p. 55-60, 2000. http:// dx.doi.org/10.1016/S0925-5214(00)00075-2.

LIMA, R. A. Z. Armazenamento de feijão: uso da embalagem a vácuo na manutenção da qualidade. 2013. 105 f. Tese (Doutorado)Universidade Federal de Lavras, Lavras, 2013.

LIMA, R. A. Z.; TOMÉ, L. M.; ABREU, C. M. P. Embalagem a vácuo: efeito no escurecimento e endurecimento do feijão durante o armazenamento. Ciência Rural, v. 44, n. 9, p. 1664-1670, 2014. http://dx.doi.org/10.1590/0103-8478cr20120832.

MANACH, C.; SCALBERT, A.; MORAND, C.; RÉMÉSY, C.; JIMENEZ, L. Polyphenols: food sources and bioavailability. The American Journal of Clinical Nutrition, v. 79, p. 727-747, 2004.

MONTEIRO, J. M.; AlBUQUERQUE, U. P.; ARAÚJO, E. L.; AMORIM, E. L. C. Taninos: uma abordagem da química à ecologia. Química Nova, v. 28, n. 5, p. 892-896, 2005.

MOUSSA, F.; KAHN, F. Uso y potencial econômico de dos palmas, Astrocaryum aculeatum Meyer y A. vulgare Martinus, em la Amazonía brasileña. In: RIOS, M.; PEDESEN, H. B. Uso y manejo de recursos vegetales. Quito: Abya-Yala, 1997. cap. 1, p. 101-116.

PRENTICE, C.; SAINZ, R. L. Cinética e deterioração apresentada por filés de carpa-capim (Ctenopharyn godonidella) embalados a vácuo sob diferentes condições de refrigeração. Ciência e Tecnologia de Alimentos, v. 25, n. 1, p. 127-131, 2005.

RAMOS, S. L. F.; MACÊDO, J. L. V.; LOPES, S. S.; RAMOS, L. F. F. Técnicas para facilitar a germinação de sementes de tucumã (Astrocaryum aculeatum Meyer). Comunicado Técnico, v. 77, p. 1-6, 2009.

RINALDI, M. M.; VIEIRA, E. A.; FIALHO, J. F.; MALAQUIAS, J. $\checkmark$. Efeito de diferentes formas de congelamento sobre raízes de mandioca. Brazilian Journal of Food Technology, v. 18, n. 2, p. 93-101, 2015. http://dx.doi.org/10.1590/1981-6723.3414.

SANTOS, R. C. O valor energético dos alimentos. Exemplo de uma determinação experimental, usando calorimetria de combustão. Química Nova, v. 33, n. 1, p. 220-224, 2010. http:// dx.doi.org/10.1590/S0100-40422010000100038.

SANTOS, T. B. A.; SILVA, N.; JUNQUEIRA, V. C. A.; PEREIRA, J. L. Microrganismos indicadores em frutas e hortaliças minimamente processadas. Brazilian Journal of Food Technology, v. 13, n. 2, p. 141-146, 2010. http://dx.doi.org/10.4260/BJFT2010130200019.

SCATOLINI-SILVA, A. M.; BORBA, H.; GIAMPIETRO-GANECO, A.; SOUZA, P. A.; BOIAGO, M. M.; MELLO, J. L. M.; VAZ, A. B. S. Qualidade física de ovos armazenados em diferentes condições de embalagens sob temperatura ambiente. Archivos de Zootecnia, v. 62, n. 238, p. 247-254, 2013. http://dx.doi. org/10.4321/S0004-05922013000200010.

SCHROTH, G.; MOTA, M. S. S.; LOPES, R.; FREITAS, A. F. Extractive use, management and in situ domestication of a weedy palm, Astrocaryumtucuma, in the central Amazon. Forest Ecology and Management, v. 202, n. 1-3, p. 161-179, 2004. http://dx.doi.org/10.1016/j.foreco.2004.07.026.

SILVA, F. A. M.; BORGES, M. F. M.; FERREIRA, M. A. Métodos para avaliação do grau de oxidação lipídica e da capacidade antioxidante. Química Nova, v. 22, n. 1, p. 94-103, 1999. http:// dx.doi.org/10.1590/S0100-40421999000100016.

SILVA, G. F. Estudo do potencial biotecnológico de Aniba canelilla (H. B. K.) Mez para obtenção de cosméticos. 2012. 119 f. Dissertação (Mestrado em Biotecnologia e Recursos Naturais da Amazônia)-Escola Superior de Ciências da Saúde, Universidade do Estado do Amazonas, Manaus, 2012.

SILVA, V. K. L.; FIGUEIREDO, R. W.; BRITO, E. S.; MAIA, G. A.; SOUSA, P. H. M.; FIGUEIREDO, E. A. T. Estabilidade da polpa do bacuri (Platonia insignis Mart.) congelada por 12 meses. Ciência e Agrotecnologia, v. 34, n. 5, p. 1293-1300, 2010.

VASCONCELOS, M. A. S.; MELO FILHO, A. B. Conservação de alimentos. Recife: EDUFRPE, 2010. 130 p. 
Estudo da conservação das propriedades nutricionais da polpa de Tucumã (Astrocaryum aculeatum) in natura em embalagens a vácuo

Azevedo, S. C. M. et al.

YUYAMA, L. K. O.; MAEDA, R. N.; PANTOJA, L.; AGUIAR, J. P. L.; MARINHO, H. A. Processamento e avaliação da vida de prateleira do Tucumã (Astrocaryum aculeatum Meyer) desidratado e pulverizado. Ciência e Tecnologia de Alimentos, v. 28, n. 2, p. 408-412, 2008.
ZANIN, E.; SILVA, I. E. C.; LOPES, T. J.; CANCELIER, A.; COSTELI, M. C.; SILVA, A. Estudo da degradação de compostos fenólicos presente em águas residuárias de postos de combustíveis utilizando fungos filamentosos (Aspergillus flavus). Revista Eletrônica em Gestão, Educação e Tecnologia Digital, v. 18, n. 1, p. 279-287, 2014. 